\title{
Self-Assembled Metal Atom Chains on Graphene Nanoribbons
}

\author{
Seon-Myeong Choi and Seung-Hoon Jhi* \\ Department of Physics, Pohang University of Science and Technology, Pohang 790-784, Korea
}

(Received 2 September 2008; published 30 December 2008)

\begin{abstract}
Electronic and magnetic properties of alkali and alkaline-earth metal doped graphene nanoribbons (GNRs) are studied by the pseudopotential density functional method. Strong site dependence is observed in metal adsorption on GNRs, and the adsorbed metal atoms are found to spontaneously form atomic chains in a particular form of GNRs. Such doped GNRs exhibit intriguing magnetic properties such as hysteresis and spin compensation as metal atoms switch from one edge to another at alternating gate voltages. Our study shows that the metal atoms can be used as reagents that can identify the edge atomic structures of GNRs and also as gate-driven spin valves that control the spin current in GNRs.
\end{abstract}

Graphene nanostructures have attracted great attention recently due to their unique and intriguing electric and transport properties [1,2]. Giant magnetoresistance [3] and quantum Hall effects [4,5] are examples that demonstrate fascinating features emerging from $2 \mathrm{D}$ electrons with linear energy dispersions in the graphene nanoribbons (GNRs). Particularly, the GNRs' large carrier mobility [1,4-7] is very promising for high-speed electronic devices $[8,9]$. Unlike carbon nanotubes, zigzag-edged graphene nanoribbons have edge-localized states, which leads to a spin-induced energy gap [10]. The electronic and transport properties are thus very sensitive to the atomic structures and chemistry of the edges. A recent measurement of the band gaps of GNRs showed width dependence but not orientation dependence [11], raising the possibility of irregular edge structures. On the other hand, previous $a b$ initio calculations showed that edge-localized states are robust against oxidation, structural defects, or mixing of different edge types [12-14]. Since the presence of edge states affects the atomic adsorption and subsequent electronic properties such as carrier density and doping characteristics, atomic adsorption on GNRs was studied using the first principles calculations to model this behavior and simulate its effect on electronic and transport properties.

Both armchair-edged GNR (AGNR) and zigzag-edged GNR (ZGNR) were studied to understand the adsorption properties, electronic structure, and magnetic properties when either an alkali metal (AM) or an alkaline-earth metal (AEM) is adsorbed. Infinitely long GNRs were simulated by the supercell method. An AGNR was chosen with a width of $25.9 \AA$ and two repeating cells along the ribbon direction. The ZGNR chosen here had a width of $27.8 \AA$ with four repeating cells along the ribbon direction. (A schematic view of the structures is shown in Figs. 1 and 2.) The edge-to-edge and layer-to-layer distance between adjacent GNRs in the supercell is 14.5 and $15.0 \AA$, respectively, which is large enough to neglect the artificial interactions between neighboring cells. Computations were carried out using the pseudopotential density func- tional method with a plane-wave basis set [15]. The exchange-correlation interactions between electrons were treated within the generalized gradient approximation as implemented by Perdew, Burke, and Ernzerhof [16]. The cutoff energy for expansion of wave functions and potentials was $400 \mathrm{eV}$, and the Monkhorst-Pack special $k$-point method was used with a grid of $1 \times 1 \times 6$ for ZGNR or with an equivalent number of $k$ points. We used the projector augmented wave pseudopotentials as provided by the Vienna ab initio software package [17]. The atomic relaxation was carried out until the Helmann-Feynman forces were less than $0.02 \mathrm{eV} / \AA$. Metal adsorption was modeled for AM ( $\mathrm{Li}, \mathrm{Na}$, and $\mathrm{K}$ ) and AEM (Be, $\mathrm{Mg}$, and $\mathrm{Ca}$ ) atoms on various sites across the GNRs.

Figure 1 summarizes our calculations of adsorption energy together with atomic structure and metal adsorption sites in AGNR. Among the top of carbon, the bridge site, and the hexagon center, metal atoms bind to the hexagon

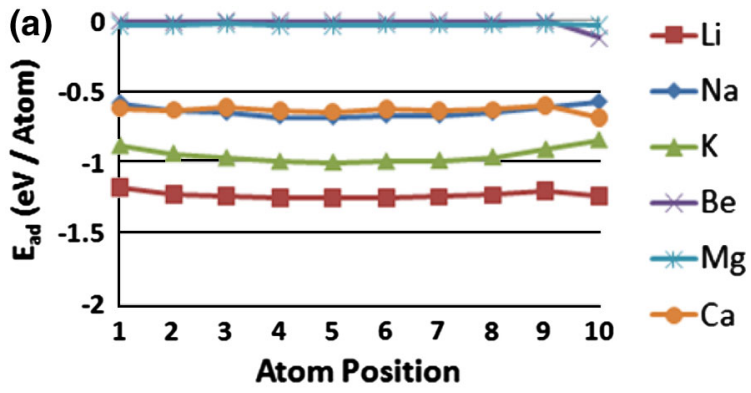

(b)

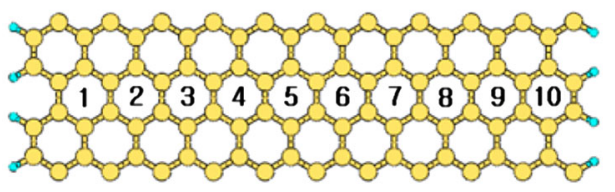

FIG. 1 (color online). (a) Calculated adsorption energy of AM $(\mathrm{Na}, \mathrm{K}$, and $\mathrm{Be}$ ) and $\mathrm{AEM}(\mathrm{Be}, \mathrm{Mg}$, and $\mathrm{Ca}$ ) atoms on an AGNR along the lateral direction. (b) The local maxima in the adsorption energy is at the top of the carbon hexagon centers enumerated from 1 to 10 . 
(a)

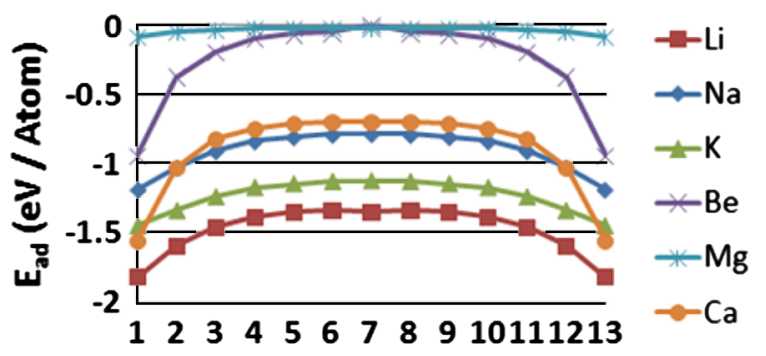

(b)

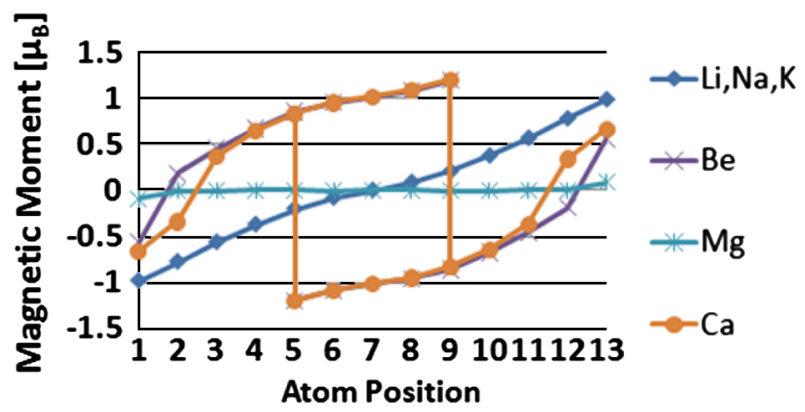

(c)

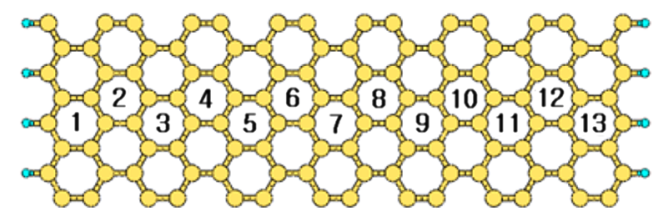

FIG. 2 (color online). (a) Calculated adsorption energy of the metal atoms on a ZGNR along the lateral direction, and (b) calculated magnetic moment of the metal-adsorbed ZGNR per unit cell shown in (c).

center most strongly. (Binding to edge hydrogen atoms is less favorable, for example, by about $0.3 \mathrm{eV}$ for $\mathrm{Na}$.) The calculated adsorption energy is correlated with the ionization energy, the radius of metal ions, and the amount of charge transfer. Simply put, metal atoms can adsorb on graphene once the electrostatic energy gain arising from charge transfer between metal and graphene is larger than the difference between the metal ionization energy and the graphene work function. Among the metal atoms considered here, Be and $\mathrm{Mg}$ do not adsorb on AGNR as they do not meet such conditions. The middle of the AGNRs provides a slightly stronger adsorption, but overall the adsorption is fairly uniform in strength along the transverse direction for all metal atoms. This is simply because the uniform charge density in AGNRs does not prefer any particular binding sites. It is found that one electron transfers from metal atoms (except $\mathrm{Be}$ and $\mathrm{Mg}$ ) to AGNR and the net magnetic moment per unit cell is either zero (AM) or $1 \mu_{B}(\mathrm{Ca})$, depending on the spin of the remaining electron in metal.

ZGNRs are particularly interesting as they have localized edges states near the Fermi level. Figure 2(a) shows the calculated binding energy of the metal atoms at various binding sites. A strong site dependence was observed in the binding energy except for $\mathrm{Mg}$, which is nonbinding due to its large ionization energy and large atomic radius. Be is also nonbinding at the center but binds to ZGNR at the edges. The binding energy is the largest at the edges and the smallest at the center for all AM and AEM atoms studied in this work. Again, our calculation shows that the binding of metal atoms to the edge hydrogen is energetically less favorable. Replacing the edge hydrogen by the metal also costs too much energy (about $3.3 \mathrm{eV}$ ). Interestingly, the adsorption energy at the center of the ZGNR is smaller than the corresponding one in AGNR. The difference is attributed to the stronger screening in AGNRs. However, the adsorption energy will converge to that on single graphenes, and the difference will become negligible when the width of GNRs is very large. The site dependence of adsorption energy on ZGNR is easily understood from the electronic structure of ZGNRs. The charge transferred from AM/AEM atoms to ZGNRs is calculated to be about one electron per metal atom and occupies the lowest-lying unoccupied edge states. The electrostatic interaction between positively charged AM/AEM atoms and ZGNRs is thus strongest at the edge and weakest in the middle. Our calculated binding energy suggests that metal atoms once absorbed on ZGNRs will readily form onedimensional atom chains at the edges. The formation of the metal atom chains (or simply Be adsorption) can be used to determine the type of graphene edge structures. The ZGNRs will show edge-localized metal atom chains, whereas the AGNR will have a rather uniform adsorption of metals. As mentioned above, localized edge states are quite robust against oxidation or structural defects and even can exist at mixed edge structures [12-14]. While the metal adsorption looks less localized in irregular edges than in clean-cut ZGNRs, our calculation suggests that the atomic adsorption can still be a useful tool to identify the edge structure. Not only AM/AEM atoms but molecules that induce significant charge transfer can also be used as chemical agents to detect the edge structure of graphenes.

Such charge transfer also affects the magnetic properties of the ZGNRs. Our spin-polarized calculations show that the configuration with opposite spin (antiferromagnetic) orientation between ferromagnetically ordered edges is more stable than the one with the same spin orientation between the two edges regardless of metal adsorption. The energy difference between the two configurations is calculated to be about $2 \mathrm{meV}$ per edge atom, consistent with previous calculations [12], and reduced to 1.5 and $0.6 \mathrm{meV}$ per edge atom, for example, for $\mathrm{Na}$ adsorption on the edges and the center, respectively. Figure 2(b) shows the calculated magnetic moment of ZGNRs with AM/AEM absorption, which also exhibits a strong site dependence. The small spin-orbit interaction [12,18] is not included here, and the sign in the magnetic moment is referred to the spin orientation designated as $\alpha$ and $\beta$ spin. Again, such site dependence is attributed to the occupational behavior of the transferred electrons. For center-site adsorption of $\mathrm{Na}$, for example, the electron occupies both left and right edge 
states evenly without any change in the magnetic moment of the ZGNRs. On the other hand, edge adsorption prefers the transferred electron to occupy the state in the same edge due to Coulomb interaction between the metal ion and charged ZGNR, which results in a net magnetic moment. If the $\mathrm{Na}$ adsorption site is shifted from the left to the right edge, the magnetic moment gradually changes from -1 to $+1 \mu_{B}$. Similar to variable resistors, AM/AEM atom adsorption can be used as a spin valve to control spin currents. Calculated magnetic moment is understood with reference to the spin polarization of injected electrons from electrodes. One possible device structure is to use a split gate to align the adsorbed metal atoms to one of the edges. This will be discussed below in more detail. The most intriguing results are obtained in the magnetic moment for AEM atom adsorption. Not only a similar site dependence but also a hysteresis is observed as atoms move from left to right edges and vice versa. The divalency of AEM atoms is responsible for such hysteresis loops. To understand such site dependence in binding energy and magnetic properties in more detail, we investigated the band structure and the electronic charge density, particularly near the Fermi level.

Figure 3 shows the calculated electronic band structures and the charge density near the Fermi level when a $\mathrm{Na}$ atom is adsorbed on the left edge. Without $\mathrm{Na}$ adsorption, the $\alpha$-spin and $\beta$-spin states are degenerate in all bands, with localized edges states of each spin occupying opposite edges due to time reversal and mirror symmetry [10]. The additional electron from $\mathrm{Na}$ adsorbed on the edge with, for example, the $\alpha$-spin electron will occupy the empty state with opposite spin ( $\beta$ spin) in that edge. Thus, the result will be a net magnetic moment of $-1 \mu_{B}$ ( $\beta$ spin). When a

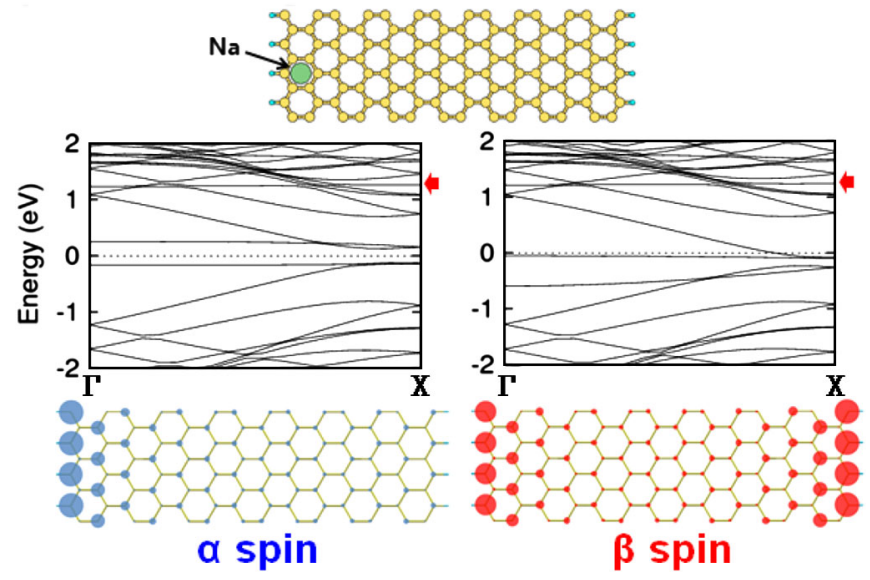

FIG. 3 (color online). Calculated band structures for $\alpha$ (left panel) and $\beta$ spin (right) of ZGNRs with $\mathrm{Na}$ atoms adsorbed on the left edge. The sodium $3 s$ level is indicated by a small red arrow. The charge density of the states near the Fermi level (with an energy window of $-0.75 \mathrm{eV}$ below the Fermi level) is shown below the band structures, with the diameter of the (blue or red online) circles denoting the density for each spin.
$\mathrm{Na}$ atom is adsorbed on the center, the net magnetic moment does not change due to equal occupation of both spin states. Other AMs (Li and $\mathrm{K}$ ) also exhibit similar adsorption properties.

The electron transfer and subsequent occupation of graphene edge states observed in monovalent AM becomes more complicated with divalent AEM atoms. According to our calculations, the valence electrons of AEM atoms, except for Mg that does not adsorb on ZGNRs, are partially transferred to ZGNRs, and the amount of the charge transfer differs from site to site. The net spin magnetic moment of AEM-adsorbed ZGNRs is the sum of each contribution from electrons in the ZGNR and in the AEM atom. The calculated net magnetic moment was used to estimate the amount of charge transfer from AEM to graphene. For Ca, it is the largest at the edge (about 1.33 electrons/Ca) and the smallest at the center $(1.00$ electron/Ca). This sitevariant charge transfer and a weak spin-spin interaction between electrons in AEM and graphene explain the hysteresis loop in calculated magnetic moments. At the edges, the charge transfer of 1.33 electrons per $\mathrm{Ca}$ atom leads to the net magnetic moment of about $-0.66 \mu_{B}$. As Ca moves to the center, its charge state gradually changes from 1.33 to 1.00. A weak spin-spin interaction between the electrons in $\mathrm{Ca}$ and in the edge prefers the same spin orientation. The transferred charge of one electron for center adsorption again occupies equally the $\alpha$ - and $\beta$-spin states of both edges; the net magnetic moment of Ca-adsorbed ZGNRs thus becomes $+1 \mu_{B}$, which is completely from the electron in $\mathrm{Ca}$. As the $\mathrm{Ca}$ atom moves to the opposite edge, the charge transfer increases to 1.33 electrons, but the spin orientation of the electron in Ca flips due to the spin interaction with the electron in the opposite edge. When the $\mathrm{Ca}$ atom moves back to the center, the total magnetic moment becomes $-1 \mu_{B}$, producing a hysteresis loop. The electrons of $\mathrm{Ca}$ in both $\alpha$-and $\beta$-spin states have the same energy (this is simply the Kramer's degeneracy) at the center, but the magnetic moment depends on the history of $\mathrm{Ca}$ atom movement along the adsorption sites. Since the adsorption energy of $\mathrm{Ca}$ at the center of ZGNRs is the smallest, however, such hysteresis of the spin magnetic moment is not expected to produce any significant effects on the magnetic properties of graphenes.

At low coverage, AM or AEM atoms are likely to adsorb on both edges with equal populations, and no significant effects on transport properties are expected except increasing carrier density. Once metal atom chains form at the edge, the net spin at both edges is very small, and the band gap induced by the magnetic ordering vanishes. Such an occupational behavior of metal at the edges, on the other hand, can be controlled by changing the chemical potential of the metal. One possible way to control the population of metal atoms at the particular edges is to apply electric fields by, for example, split gates. In order to assess this possibility, we studied the ZGNRs with metal atoms ad- 


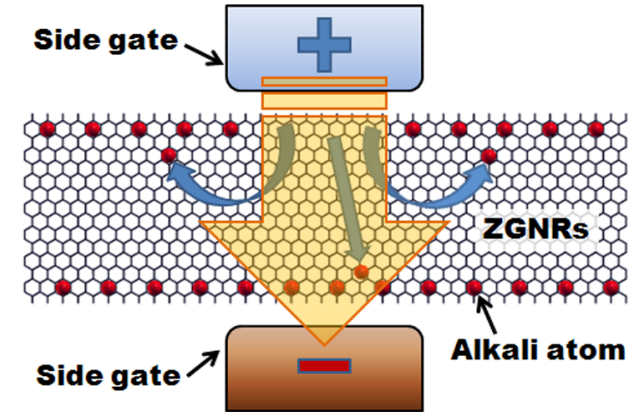

(a)

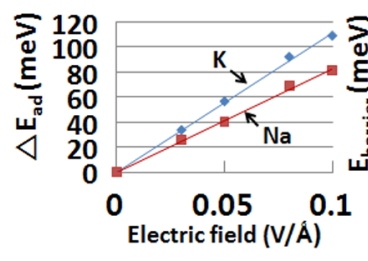

(b)

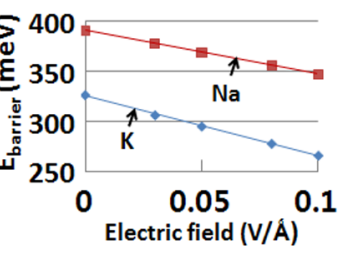

(C)
FIG. 4 (color online). (a) A schematic drawing of a spin-valve structure with a side gate that drives AEM atoms from one edge of a ZGNR to the other. (b) and (c) show the calculated changes in adsorption energy and the (transverse) energy barrier across the edges at varying gate electric fields, respectively. Calculated energies scale linearly with the applied gate voltage within the range considered.

sorbed on both edges with or without external fields. Figure 4 shows a schematic of a spin-valve device structure and the calculated results of binding energy variation upon applied gate voltage. In order to simulate the split-gate electric field, we applied a sawtooth-type potential across the ZGNR. When metal atoms are adsorbed on graphene, the kinetic barrier for metal atoms to move from one adsorption site to the next is calculated to be about 4,7 , and $127 \mathrm{meV}$ for $\mathrm{K}, \mathrm{Ca}$, and $\mathrm{Na}$, respectively. In the middle of nanoribbons, metal atoms can thus migrate fairly smoothly. From the edges to the center, $\mathrm{K}$ atoms experience an energy barrier of $327 \mathrm{meV}$, which is simply the difference in binding energy at the center and at the edge. At a gate electric field of about $0.1 \mathrm{~V} / \mathrm{A}$, one edge has larger adsorption energy by $109 \mathrm{meV}$ than the other and the barrier is reduced to $266 \mathrm{meV}$. As both $\Delta E_{\mathrm{ad}}$ and $E_{\text {barrier }}$ are linear in the gate electric field, the metal atoms are expected to migrate easily at a few tenths of $\mathrm{V} / \AA$. The metal migration from one edge to another can be controlled by applying a gate voltage and by heating the substrate. For practical applications in spin-valve devices [19-22], our calculations show that $\mathrm{K}$ is better than $\mathrm{Na}$ as it is more sensitive to gate voltage both in adsorption energy and in the energy barrier. $\mathrm{K}$ is known to readily form a fluidlike phase on graphite at low coverage even at low temperatures
$[23,24]$, whereas $\mathrm{Na}$ forms islands on graphite at temperatures of $90 \mathrm{~K}$ or less $[24,25]$.

In summary, we studied the AM and AEM adsorption on graphene nanoribbons. It was found that the metal atoms prefer edge absorption in ZGNRs, but they do not show such a tendency in AGNRs. Such a preference implies the spontaneous formation of one-dimensional atom chains at the edges. The spin magnetic moment upon metal absorption also shows such site dependence, which leads to hysteresis in the magnetic moment. Spin-valve device structures operated by side gates were proposed utilizing the site dependence of metal absorption.

This work is supported by the Korea Research Foundation Grant funded by Korea Government (MOEHRD, Basic Research Promotion Fund, Grant No. KRF-2005-070-C00041).

*jhish@postech.ac.kr

[1] K. S. Novoselov et al., Science 306, 666 (2004).

[2] A. K. Geim and K.S. Novoselov, Nature Mater. 6, 183 (2007).

[3] W. Y. Kim and K. S. Kim, Nature Nanotech. 3, 408 (2008).

[4] Y. B. Zhang et al., Nature (London) 438, 201 (2005).

[5] K. S. Novoselov et al., Nature (London) 438, 197 (2005).

[6] K.S. Novoselov et al., Proc. Natl. Acad. Sci. U.S.A. 102, 10451 (2005).

[7] E. H. Hwang, S. Adam, and S. Das Sarma, Phys. Rev. Lett. 98, 186806 (2007).

[8] C. Berger et al., Science 312, 1191 (2006).

[9] M. C. Lemme et al., IEEE Electron Device Lett. 28, 282 (2007).

[10] Y. W. Son, M. L. Cohen, and S. G. Louie, Phys. Rev. Lett. 97, 216803 (2006).

[11] M. Y. Han et al., Phys. Rev. Lett. 98, 206805 (2007).

[12] Y. W. Son, M. L. Cohen, and S. G. Louie, Nature (London) 444, 347 (2006).

[13] K. Nakada, M. Fujita, G. Dresselhaus, and M.S. Dresselhaus, Phys. Rev. B 54, 17954 (1996).

[14] O. Hod, V. Barone, J. E. Peralta, and G. E. Scuseria, Nano Lett. 7, 2295 (2007).

[15] M. L. Cohen, Phys. Scr. T1, 5 (1982).

[16] J. P. Perdew, K. Burke, and M. Ernzerhof, Phys. Rev. Lett. 77, 3865 (1996).

[17] G. Kresse and J. Hafner, Phys. Rev. B 49, 14251 (1994).

[18] G. Dresselhaus, M. Dresselhaus, and J. G. Mavrodies, Carbon 4, 433 (1966).

[19] E. W. Hill et al., IEEE Trans. Magn. 42, 2694 (2006).

[20] M. Nishioka and A. M. Goldman, Appl. Phys. Lett. 90, 252505 (2007).

[21] S. J. Cho, Y. F. Chen, and M. S. Fuhrer, Appl. Phys. Lett. 91, 123105 (2007).

[22] N. Tombros et al., Nature (London) 448, 571 (2007).

[23] K. M. Hock and R. E. Palmer, Surf. Sci. 284, 349 (1993).

[24] M. Caragiu and S. Finberg, J. Phys. Condens. Matter 17, R995 (2005).

[25] M. Breitholtz et al., Phys. Rev. B 67, 235416 (2003). 